\title{
DEEP INELASTIC SCATTERING: EXPERIMENTS ON THE PROTON AND THE OBSERVATION OF SCALING
}

\author{
Nobel Lecture, December 8, 1990
}

by

HENRY W. KENDALL

Massachusetts Institute of Technology, Cambridge, Massachusetts, USA

\section{Introduction}

\section{A. Overview of the Electron Scattering Program}

In late 1967 the first of a long series of experiments on highly inelastic electron scattering was started at the two mile accelerator at the Stanford Linear Accelerator Center (SLAC) using liquid hydrogen and, later, liquid deuterium targets. Carried out by a collaboration from the Massachusetts Institute of Technology (MIT) and SLAC, the object was to look at large energy loss scattering of electrons from the nucleon (the generic name for the proton and neutron), a process soon to be dubbed deep inelastic scattering. Beam energies up to $21 \mathrm{GeV}$, the highest electron energies then available, and large electron fluxes, made it possible to study the nucleon to very much smaller distances than had previously been possible. Because quantum electrodynamics provides an explicit and well-understood description of the interaction of electrons with charges and magnetic moments, electron scattering had, by 1968 , already been shown to be a very powerful probe of the structures of complex nuclei and individual nucleons.

H ofstadter and his collaborators had discovered, by the mid-1960s that as the momentum transfer in the scattering increased, the scattering cross section dropped sharply relative to that from a point charge. The results showed that nucleons were roughly $10^{-13} \mathrm{~cm}$ in size, implying a distributed structure. The earliest MIT-SLAC studies, in which California Institute of Technology physicists also collaborated, looked at elastic electron-proton scattering, later ones at electro-production of nucleon resonances with excitation energies up to less than $2 \mathrm{GeV}$. Starting in 1967, the MIT-SLAC collaboration employed the higher electron energies made available by the newly completed SLAC accelerator to continue such measurements, before beginning the deep inelastic program.

Results from the inelastic studies arrived swiftly: the momentum transfer dependence of the deep inelastic cross sections was found to be weak, and the deep inelastic form factors - which embodied the information about the proton structure - depended unexpectedly only on a single variable rather than the two allowed by kinematics alone. These results were inconsistent with the current expectations of most physicists at the time. The 
general belief had been that the nucleon was the extended object found in elastic electron scattering but with the diffuse internal structure seen in pion and proton scattering. The new experimental results suggested pointlike constituents but were puzzling because such constituents seemed to contradict well-established beliefs. Intense interest in these results developed in the theoretical community and, in a program of linked experimental and theoretical advances extending over a number of years, the internal constituents were ultimately identified as quarks, which had previously been devised in 1964 as an underlying, quasi-abstract scheme to justify a highly successful classification of the then-known hadrons. This identification opened the door to development of a comprehensive field theory of hadrons (the strongly interacting particles), called Quantum Chromodynamics (QCD), that replaced entirely the earlier picture of the nucleons and mesons. QCD in conjunction with electroweak theory, which describes the interactions of leptons and quarks under the influence of the combined weak and electromagnetic fields, constitutes the Standard Model, all of whose predictions, at this writing, are in satisfactory agreement with experiment. The contributions of the MIT-SLAC inelastic scattering program were recognized by the award of the $1990 \mathrm{~N}$ obel Prize in Physics.

\section{B. Organization of lectures}

There are three lectures that, taken together, describe the MIT-SLAC experiments. The first, written by R.E.Taylor (Reference I), sets out the early history of the construction of the two mile accelerator, the proposals made for the construction of the electron scattering facility, the antecedent physics experiments at other laboratories, and the first of our scattering experiments which determined the elastic proton structure form factors. This paper describes the knowledge and beliefs about the nucleon's internal structure in 1968, including the conflicting views on the validity of the quark model and the "bootstrap" models of the nucleon. This is followed by a review of the inelastic scattering program and the series of experiments that were carried out, and the formalism and variables. Radiative corrections are described and then the results of the inelastic electron-proton scattering measurements and the physics picture - the naive parton model - that emerged. The last lecture, by J. I. Friedman (Reference 2), is concerned with the later measurements of inelastic electron-neutron and electron-proton measurements and the details of the physical theory - the constituent quark model - which the experimental scattering results stimulated and subsequently, in conjunction with neutrino studies, confirmed.

\section{Nucleon and Hadronic Structure in 1968}

At the time the MIT-SLAC inelastic experiments started in 1968, there was no detailed model of the internal structures of the hadrons. Indeed, the very notion of "internal structure" was foreign to much of the then-current theory. Theory attempted to explain the soft scattering - that is, rapidly 
decreasing cross sections as the momentum transfer increased - which was the predominant characteristic of the high energy hadron-hadron scattering data of the time, as well as the hadron resonances, the bulk of which were discovered in the late 1950s and 1960s. Quarks had been introduced, quite successfully, to explain the static properties of the array of hadrons. Nevertheless, the available information suggested that hadrons were "soft" inside, and would yield primarily distributions of scattered electrons reflecting diffuse charge and magnetic moment distributions with no underlying point-like constituents. Quark constituent models were gleams in the eyes of a small handful of theorists, but had serious problems, then unsolved, which made them widely unpopular as models for the high energy interactions of hadrons.

The need to carry out calculations with forces that were known to be very strong introduced intractable difficulties: perturbation theory, in particular, was totally unjustified. This stimulated renewed attention to S-matrix theory (Reference 3), an attempt to deal with these problems by consideration of the properties of a matrix that embodied the array of strong interaction transition amplitudes from all possible initial states to all possible final states.

\section{A. Theory: Nuclear Democracy}

An approach to understanding hadronic interactions, and the large array of hadronic resonances, was the bootstrap theory (Reference 4), one of several elaborations of S-matrix theory. It assumed that there were no "fundamental" particles: each was a composite of the others. Sometimes referred to as "nuclear democracy," the theory was at the opposite pole from constituent theories.

Regge theory (Reference 5), a very successful phenomenology, was one elaboration of S-matrix theory which was widely practiced. Based initially on a new approach to non-relativistic scattering, it was extended to the relativistic S-matrix applicable to high energy scattering (Reference 6). The known hadrons were classified according to which of several "trajectories" they lay on. It provided unexpected connections between reactions at high energies to resonances in the crossed channels, that is, in disconnected sets of states. For scattering, Regge theory predicted that at high energy, hadron-hadron scattering cross sections would depend smoothly on $\mathrm{s}$, the square of the center of mass energy, as $A(s) \sim \mathrm{s}^{(\alpha(0))}$, and would fall exponentially with $t$, the square of the space-like momentum transfer, as

$$
\mathrm{A}(\mathrm{t}) \sim \exp \left(\alpha^{\prime} \mathrm{t} \ln \left(\mathrm{s} / \mathrm{s}_{0}\right)\right)
$$

Regge theory led to duality, a special formulation of which was provided by Veneziano's dual resonance model (Reference 7). These theories still provide the best description of soft, low momentum transfer scattering of pions and nucleons from nucleons, all that was known in the middle 1960s. There was a tendency, in this period, to extrapolate these low momentum transfer results so as to conclude there would be no hard scattering at all. 
S-matrix concepts were extended to the electromagnetic processes involving hadrons by the Vector Meson Dominance (VMD) model (Reference 8). A ccording to VMD, when a real or virtual photon interacts with a hadron, the photon transforms, in effect, into one of the low mass vector mesons that has the same quantum numbers as the photon (primarily the rho, omega and phi mesons). In this way electromagnetic amplitudes were related to hadronic collision amplitudes, which could be treated by S-matrix methods. The VMD model was very successful in phenomena involving real photons and many therefore envisaged that VMD would also deal successfully with the virtual photons exchanged in inelastic electron scattering. Naturally, this also led to the expectation that electron scattering would not reveal any underlying structure.

All of these theories, aside from their applications to hadron-hadron scattering and the properties of resonances, had some bearing on nucleon structure as well, and were tested against the early MIT-SLAC results.

\section{B. Quark Theory of 1964}

The quark ${ }^{1}$ was born in a 1964 paper by Murray Gell-Mann (Reference 9) and, independently, by George Zweig (Reference 10). For both, the quark (a term Zweig did not use until later) was a means to generate the symmetries of SU(3), the "Eightfold Way," Gell-Mann and Ne'emann's (Reference 11) highly successful 1961 scheme for classifying the hadrons. Combinations of spin I/ 2 quarks, with fractional electric charges, and other appropriate quantum numbers, were found to reproduce the multiplet structures of all the observed hadrons. Fractional charges were not necessary but provided the most elegant and economical scheme. Three quarks were required for baryons, later referred to as "valence" quarks, and quarkantiquark pairs for mesons. Indeed the quark picture helped solve some difficulties with the earlier symmetry groupings (Reference 12). The initial successes of the theory stimulated numerous free quark searches. There were attempts to produce them with accelerator beams, studies to see if they were produced in cosmic rays, and searches for "primordial" quarks by Millikan oil drop techniques sensitive to fractional charges. None of these has ever been successful (Reference 13).

\section{Constituent Quark Picture}

There were serious problems in having quarks as physical constituents of nucleons and these problems either daunted or repelled the majority of the theoretical community, including some of its most respected members (Reference 14). The idea was distasteful to the S-matrix proponents. The problems were, first, that the failure to produce quarks had no precedent in

1 The word quork was invented by Murray Cell-Mann, who later found quark in the novel Finnegan's Wake, by James Joyce, and adopted what has become the accepted spelling. Joyce apparently employed the word as a corruption of the word quart. The author is grateful to Murray Gell-Mann for a discussion clarifying the matter. 
physicists' experience. Second, the lack of direct production required the quarks to be very massive, which, for the paired quark configurations of the mesons, meant that the binding had to be very great, a requirement that led to predictions inconsistent with hadron-hadron scattering results. Third, the ways in which they were combined to form the baryons, meant that they could not obey the Pauli exclusion principle, as required for spin one-half particles. Fourth, no fractionally charged objects had ever been unambiguously identified. Such charges were very difficult for many to accept, for the integer character of elementary charges was long established. Enterprising theorists did construct quark theories employing integrally charged quarks, and others contrived ways to circumvent the other objections. Nevertheless, the idea of constituent quarks was not accepted by the bulk of the physics community, while others sought to construct tests that the quark model was expected to fail (Reference 15).

Some theorists persisted, nonetheless. Dalitz (Reference 16) carried out complex calculations to help explain not only splittings between hadron multiplets but the splittings within them also, using some of the theoretical machinery employed in nuclear spectroscopy calculations. Calculations were carried out on other aspects of hadron dynamics, for example, the successful prediction that $\Delta^{+}$decay would be predominantly magnetic dipole (Reference 17). Owing to the theoretical difficulties just discussed, the acceptance of quarks as the basis of this successful phenomenology was not carried over to form a similar basis for high energy scattering.

Gottfried studied electron-proton scattering with a model assuming point quarks, and argued that it would lead to a total cross section (elastic plus inelastic) at fixed momentum transfer, identical to that of a point charge, but he expressed great skepticism that this would be borne out by the forthcoming data (Reference 18). With the exception of Gottfried's work and one by Bjorken stimulated by current algebra, discussed below, all of the published constituent quark calculations were concerned with low energy processes or hadron characteristics rather than high energy interactions. Zweig carried out calculations assuming that quarks were indeed hadron constituents but his ideas were not widely accepted (Reference 19).

Thus, one sees that the tide ran against the constituent quark model in the 60 s (Reference 20). One reviewer's summary of the style of the 60s was that "quarks came in handy for coding information but should not be taken seriously as physical objects" (Reference 21). While quite helpful in low energy resonance physics, it was for some "theoretically disreputable," and was felt to be largely peripheral to a description of high energy soft scattering (Reference 22).

\section{Current Algebra}

Following his introduction of quarks, Gell-Mann, and others, developed "current algebra," which deals with hadrons under the influence of weak and electromagnetic interactions. Starting with an assumption of free quark fields, he was able to find relations between weak currents that reproduced 
the current commutators postulated in constructing his earlier hadronic symmetry groups. Current algebra had become very important by 1966 . It exploited the concept of local observables - the current and charge densities of the weak and electromagnetic interactions. These are field theoretic in character and could only be incorporated into S-matrix cum bootstrap theory by assumptions like VMD. The latter are plausible for moderate momentum transfer, but hardly for transfer large compared to hadron masses. As a consequence, an important and growing part of the theoretical community was thinking in field theoretic terms.

Current algebra also gave rise to a small but vigorous "sum rule" industry. Sum rules are relationships involving weighted integrals over various combinations of cross sections. The predictions of some of these rules were important in confirming the deep inelastic electron and neutrino scattering results, after these became available (Reference 23 ).

Gell-Mann made clear that he was not suggesting that hadrons were made up of quarks (Reference 24), although he kept open the possibility that they might exist (Reference 25). Nevertheless, current algebra reflected its constituent-quark antecedents, and Bjorken used it to demonstrate that sum rules derived by him and others required large cross sections for these to be satisfied. He then showed that such cross sections arose naturally in a quark constituent model (Reference 26), in analog to models of nuclei composed of constituent protons and neutrons, and also employed it to predict the phenomena of scaling, discussed at length below. Yet Bjorken and others were at a loss to decide how the point-like properties that current algebra appeared to imply were to be accommodated (Reference 27).

\section{E. Theoretical Input to The Scattering Program}

In view of the theoretical situation as set out above, there was no consideration that a possible point-like substructure of the nucleon might be observable in electron scattering during the planning and design of the electron scattering facility. Deep inelastic processes were, however, assessed in preparing the proposal submitted to SLAC for construction of the facility (Reference 28). Predictions of the cross sections employed a model assuming off-mass-shell photo-meson production, using photoproduction cross sections combined with elastic scattering structure functions, in what was believed to be the best guide to the yields expected. These were part of extensive calculations, carried out at MIT, designed to find the magnitude of distortions of inelastic spectra arising from photon radiation, necessary in planning the equipment and assessing the difficulty of making radiative corrections. It was found ultimately that these had underpredicted the actual yields by between one and two orders of magnitude.

\section{The Scattering Program}

The linear accelerator that provided the electron beam employed in the inelastic scattering experiments was, and remains to the date of this paper, a 


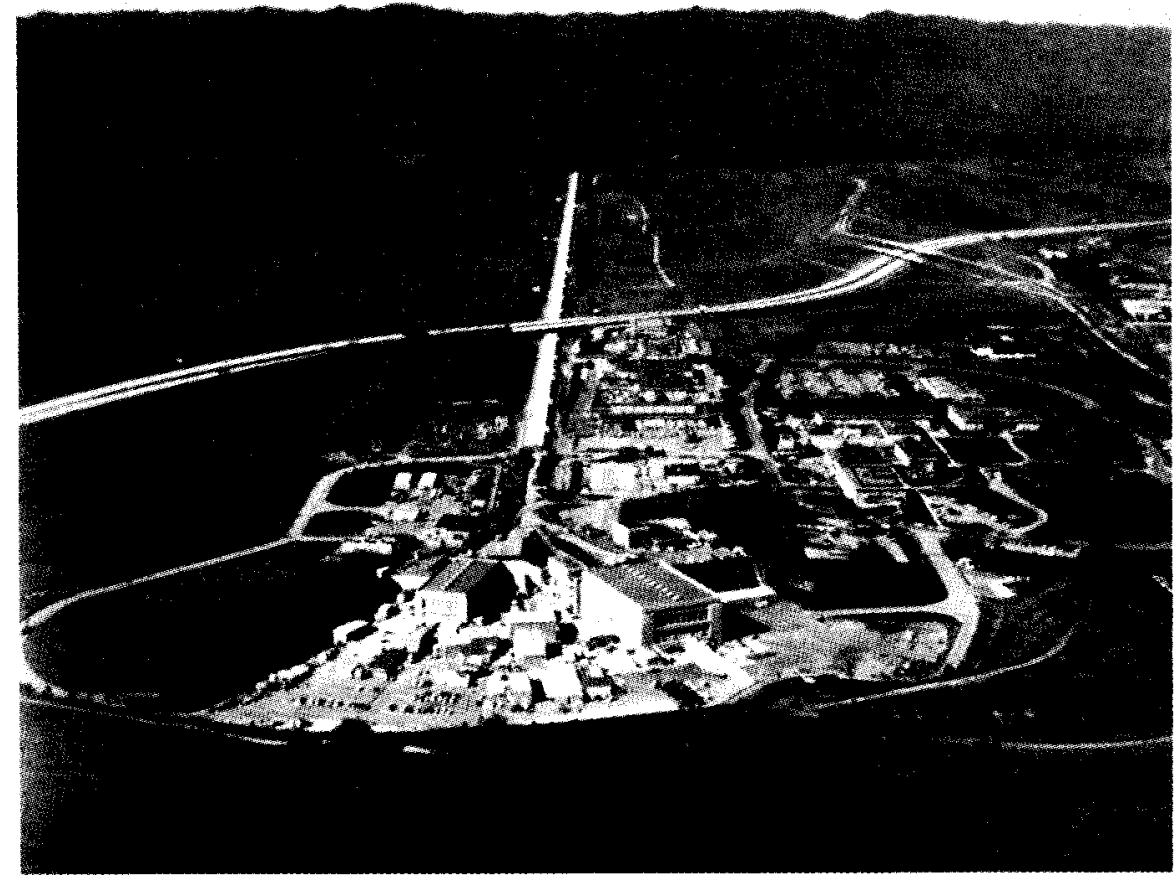

Fig. 1. View of the Stanford Linear Accelerator. The electron injector is at the top, the experimental area in lower center. The deep inelastic scattering studies were carried out in End Station A, the largest of the buildings in the experimental area.

device unique among high energy particle accelerators. See Figure 1. An outgrowth of the smaller, $1 \mathrm{GeV}$ accelerator employed by Hofstadter in his studies of the charge and magnetic moment distributions of the nucleon, it relied on advanced klystron technology devised by Stanford scientists and engineers to provide the high levels of microwave power necessary for onepass acceleration of electrons. Proposed in 1957, approved by the Congress in 1962, its construction was initiated in 1963. It went into operation in 1967, on schedule, having cost \$114M (Reference 29).

The experimental collaboration began in 1964. After 1965, R. E. Taylor was head of SLAC Group A with J.I.Friedman and the present author sharing responsibility for the M.I.T. component. A research group from California Institute of Technology joined in the construction cycle and the elastic studies but withdrew before the inelastic work started in order to pursue other interests.

The construction of the facility to be employed in electron scattering was nearly concurrent with the accelerator's construction. This facility was large for its time. A $200 \mathrm{ft}$. by $125 \mathrm{ft}$. shielded building housed three magnetic spectrometers with an adjacent "counting house" containing the fast electronics and a computer, also large for its time, where experimenters controlled the equipment and conducted the measurements. See Figure 2a and $2 \mathrm{~b}$. The largest spectrometer would focus electrons up to $20 \mathrm{GeV}$ and was employed at scattering angles up to $10^{\circ}$. A second spectrometer, useful to 8 



Fig. 2. (a) Plan view of End Station $A$ and the two principal magnetic spectrometers employed for analysis of scattered electrons. (b) Configuration of the $8 \mathrm{GeV}$ spectrometer, employed at scattering angles greater than $12^{\circ}$. 
$\mathrm{GeV}$, was used initially out to $34^{\circ}$, and a third, focusing to $1.6 \mathrm{GeV}$, constructed for other purposes, was employed in one set of large angle measurements to help determine the uniformity in density of the liquified target gases. The detectors were designed to detect only scattered electrons. The very short duty cycle of the pulsed beam precluded studying the recoil systems in coincidence with the scattered electrons: it would have given rise to unacceptable chance coincidence rates, swamping the signal.

The elastic studies started in early 1967 with the first look at inelastic processes from the proton late the same year. By the spring of 1968, the first inelastic results were at hand. The data were reported at a major scientific meeting in Vienna in August and published in 1969 (Reference 30). Thereafter, a succession of experiments were carried out, most of them, from 1970 on, using both deuterium and hydrogen targets in matched sets of measurements so as to extract neutron scattering cross sections with a minimum of systematic error. These continued well into the 1970s. One set of measurements (Reference 31) studied the atomic-weight dependence of the inelastic scattering, primarily at low momentum transfers, studies that were extended to higher momentum transfers in the early 1980s, and involved extensive reanalysis of earlier MIT-SLAC data on hydrogen, deuterium and other elements (Reference 32).

The collaboration was aware from the outset of the program that there were no accelerators in operation, or planned, that would be able to confirm the entire range of results. The group carried out independent data analyses at MIT and at SLAC to minimize the chance of error. One consequence of the absence of comparable scattering facilities was that the collaboration was never pressed to conclude either data taking or analysis in competitive circumstances. It was possible throughout the program to take the time necessary to complete work thoroughly.

\section{Scattering Formalism and Radiative Corrections}

\section{A. Fundamental Processes}

The relation between the kinematic variables in elastic scattering, as shown in Figure 3, is:

$$
v=E-E^{\prime}=q^{2} /(2 M) \quad q^{2}=2 E E^{\prime}(1-\cos \theta)
$$

where $E$ is the initial and $E$ ' the final electron energy, $\theta$ the laboratory angle of scattering, $v$ the electron energy loss, $q$ the four-momentum transferred to the target nucleon, and $M$ the proton mass.

The cross section for elastic electron-proton scattering has been calculated by Rosenbluth (Reference 33) in first Born approximation, that is, to leading order in $\alpha=$ l/ 137:

$$
\frac{d \sigma}{d \Omega}(E)=\sigma_{\mathrm{M}}(E)\left\{\frac{E^{\prime}}{E}\right\}\left\{\frac{\mathrm{G}_{\mathrm{Ep}}^{2}\left(q^{2}\right)+\tau G_{\mathrm{Mp}}^{2}\left(q^{2}\right)}{1+\tau}+2 \tau G_{\mathrm{Mp}}^{2} \tan ^{2}(\theta / 2)\right\}
$$




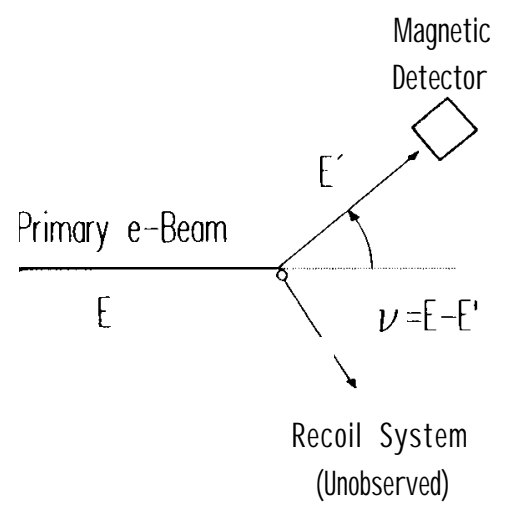

Fig. 3. Scattering kinematics.

where

$$
\sigma_{\mathrm{M}}=\frac{4 a^{2} E^{\prime 2}}{q^{4}} \cos ^{2}\left(\frac{\theta}{2}\right)
$$

is the Mott cross section for elastic scattering from a point proton, and

$$
\tau=q^{2} /\left(4 M^{2}\right)
$$

In these equations, and in what follows, $\hbar=\mathrm{c}=1$, and the electron mass has been neglected. The functions $G_{\mathrm{Ep}}\left(q^{2}\right)$ and $G_{\mathrm{Mp}}\left(q^{2}\right)$, the electric and magnetic form factors, respectively, describe the time-averaged structure of the proton. In the non-relativistic limit the squares of these functions are the Fourier transforms of the spatial distributions of charge and magnetic moment, respectively. As can be seen from Equation (2) magnetic scattering is dominant at high $q^{2}$. Measurements (Reference 34) show that $\mathrm{G}_{\mathrm{Mp}_{\mathrm{p}}}$ is roughly described by the "dipole" approximation:

$$
G_{\mathrm{Mp}} / \mu=1 /\left(1+q^{2} / 0.71\right)^{2}
$$

where $q^{2}$ is measured in $(\mathrm{GeV})^{2}$ and $\mu=2.79$ is the proton's magnetic moment. Thus, at large $q^{2}$ an additional $1 / q^{3}$ dependence beyond that of $\sigma_{\mathrm{M}}$ is imposed on the elastic scattering cross section as a consequence of the finite size of the proton. This is shown in Figure 4.

In inelastic scattering, energy is imparted to the hadronic system. The invariant or missing mass $\mathrm{W}$ is the mass of the final hadronic state. It is given by:

$$
W^{2}=\left(2 M v+M^{2}-q^{2}\right)
$$

When only the electron is observed the composition of the hadronic final state is unknown except for its invariant mass $W$. On the assumption of one photon exchange (Figure 5), the differential cross section for electron scattering from the nucleon target is related to two structure functions $W_{1}$ and $W_{2}$ according to (Reference 35): 


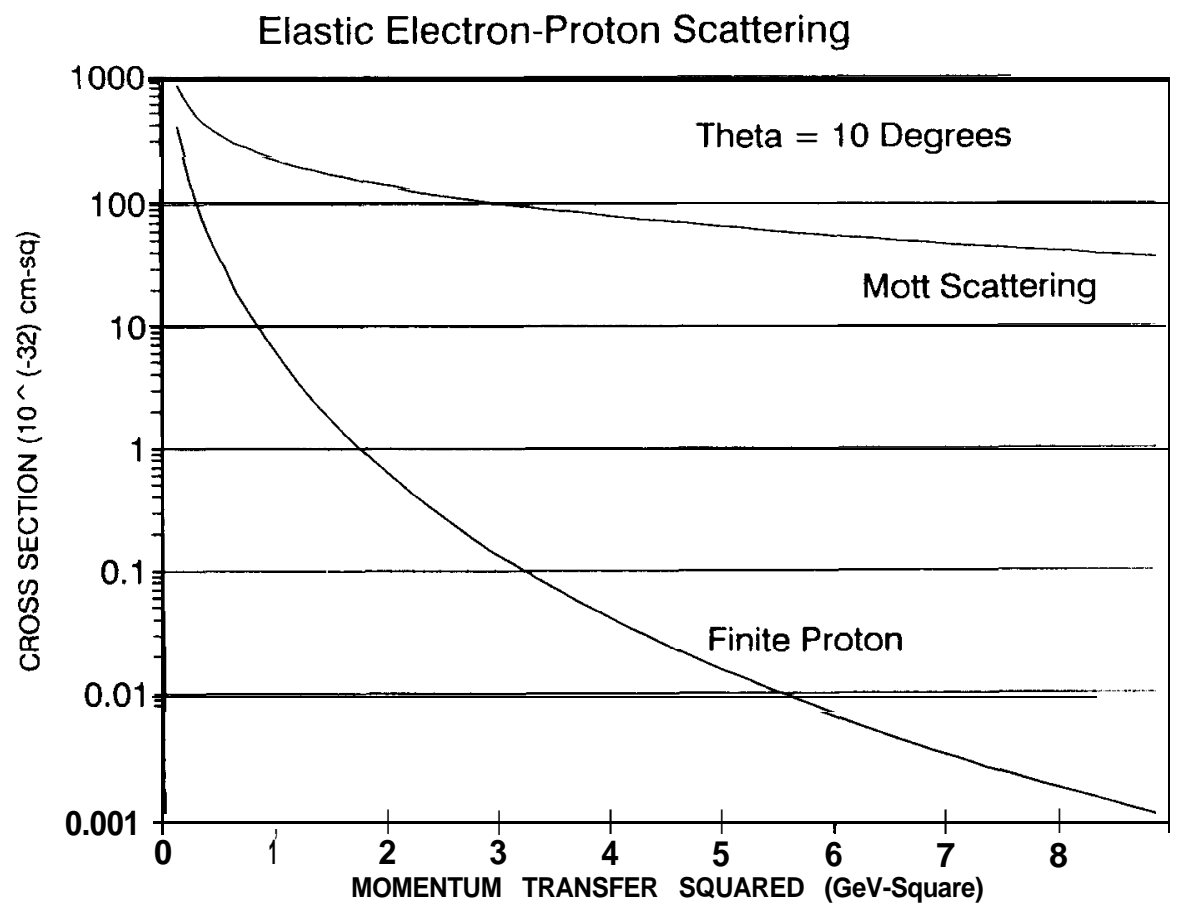

Fig. 4. Elastic scattering cross sections for electrons from a "point" proton and for the actual proton. The differences are attributable to the finite sire of the proton.

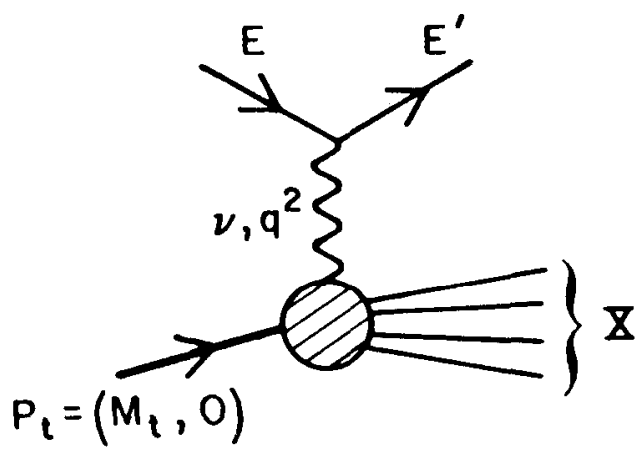

Fig. 5. Feynman diagram for inelastic electron scattering.

$$
\frac{d^{2} \sigma}{d \Omega d E^{\prime}}\left(E, E^{\prime}, \theta\right)=\sigma_{\mathrm{M}}\left[W_{2}\left(v, q^{2}\right)+2 W_{1}\left(v, q^{2}\right) \tan ^{2}(\theta / 2)\right]
$$

This expression is the analog of the Rosenbluth cross section given above. The structure functions $W_{1}$ and $W_{2}$ are similarly defined by Equation (3) for the proton, deuteron, or neutron; they summarize all the information about the structure of the target particles obtainable by scattering unpolarized electrons from an unpolarized target.

Within the single-photon-exchange approximation, one may view inelas- 
tic electron scattering as photoproduction by "virtual" photons. Here, as opposed to photoproduction by real photons, the photon mass $q^{2}$ is variable and the exchanged photon may have a longitudinal as well as a transverse polarization. If the final state hadrons are not observed, the interference between these two components averages to zero, and the differential cross section for inelastic electron scattering is related to the total cross sections for absorption of transverse, $\sigma_{\mathrm{T}}$, and longitudinal, $\sigma_{\mathrm{L}}$, virtual photons according to (Reference 36)

$$
\frac{d^{2} \sigma}{d \Omega d E^{\prime}}\left(E, E^{\prime}, \theta\right)=\Gamma\left[\sigma_{\mathrm{T}}\left(v, q^{2}\right)+\varepsilon \sigma_{\mathrm{L}}\left(v, q^{2}\right)\right]
$$

where

$$
\begin{gathered}
\Gamma=\frac{a}{4 \pi^{2}} \frac{K E^{\prime}}{q^{2} E}\left(\frac{2}{1-\varepsilon}\right) \\
\varepsilon=\left[1+2\left(1+v^{2} / q^{2}\right) \tan ^{2}(\theta / 2)\right]^{-1}
\end{gathered}
$$

and

$$
K=\left(W^{2}-M^{2}\right) /(2 M)
$$

The quantity $\Gamma$ is the flux of transverse virtual photons and $\varepsilon$ is the degree of longitudinal polarization. The cross sections $\sigma_{\mathrm{T}}$ and $\sigma_{\mathrm{L}}$ are related to the structure functions $W_{1}$ and $W_{2}$ by

$$
\begin{aligned}
& W_{1}\left(v, q^{2}\right)=\frac{K}{4 \pi^{2} a} \sigma_{\mathrm{T}}\left(v, q^{2}\right) \\
& W_{2}\left(v, q^{2}\right)=\frac{K}{4 \pi^{2} a} \quad\left(\frac{q^{2}}{q^{2}+v^{2}}\right) \quad\left[\sigma_{\mathrm{T}}\left(v, q^{2}\right)+\sigma_{\mathrm{L}}\left(v, q^{2}\right)\right]
\end{aligned}
$$

In the limit $q^{2} \rightarrow 0$, gauge invariance requires that $\sigma_{\mathrm{L}} \rightarrow 0$ and $\sigma_{\mathrm{T}} \rightarrow \sigma_{\gamma}(\mathrm{v})$, where $\sigma_{\gamma}(v)$ is the photoproduction cross section for real photons. The quantity $R$, defined as the ratio $\sigma_{\mathrm{L}} / \sigma_{\mathrm{T}}$ is related to the structure functions by

$$
R\left(v, q_{2}\right) \equiv \sigma_{\mathrm{L}} / \sigma_{\mathrm{T}}=\left(W_{2} / W_{1}\right)\left(1+v^{2} / q^{2}\right)-1
$$

A separate determination of the two inelastic structure functions $W$, and $W_{2}$ (or, equivalently, $\sigma_{\mathrm{L}}$ and $\sigma_{\mathrm{T}}$ ) requires values of the differential cross section at several values of the angle $\sigma$ for fixed $v$ and $q^{2}$. According to Equation (4) $\sigma_{\mathrm{L}}$ is the slope and $\sigma_{\mathrm{T}}$ is the intercept of a linear fit to the quantity $\Sigma$ where:

$$
\Sigma=\frac{1}{\Gamma} \frac{d^{2} \sigma}{d \Omega d E^{\prime}}\left(v, q^{2}, \theta\right)
$$

The structure functions $W$, and $W_{2}$ are then directly calculable from Eq. (5). Alternatively, one can extract $W$, and $W_{2}$ from a single differential crosssection measurement by inserting a particular functional form for $R$ in the equations 


$$
\begin{aligned}
& W_{1}=\frac{1}{\sigma_{\mathrm{M}}} \frac{d^{2} \sigma}{d \Omega d E^{\prime}}\left[(1+R)\left(\frac{q^{2}}{q^{2}+v^{2}}\right)+2 \tan ^{2}\left(\frac{\theta}{2}\right)\right]^{-1} \\
& W_{2}=\frac{1}{\sigma_{\mathrm{M}}} \frac{d^{2} \sigma}{d \Omega d E^{\prime}}\left[1+\left(\frac{2}{1+R}\right)\left(\frac{q^{2}+v^{2}}{q^{2}}\right) \tan ^{2}\left(\frac{\theta}{2}\right)\right]^{-1}
\end{aligned}
$$

Equations (5) through (7) apply equally well for the proton, deuteron, or neutron.

In practice, it was convenient to determine values of $\sigma_{\mathbf{L}}$ and $\sigma_{\mathrm{T}}$ from straight line fits to differential cross sections as functions of $\boldsymbol{\varepsilon} . R$ was determined from the values of $\sigma_{\mathrm{L}}$ and $\sigma_{\mathrm{T}}$, and $\mathrm{W}_{1}$ and $W_{2}$ were, as shown above, determined from $R$.

\section{B. Scale Invariance and Scaling Variables.}

By investigating models that satisfied current algebra, Bjorken (Reference 37) had conjectured that in the limit of $q^{2}$ and $v$ approaching infinity, with the ratio $\omega=2 \mathrm{Mv} / \mathrm{q}^{2}$ held fixed, the two quantities $\mathrm{vW}_{2}$ and $\mathrm{W}_{1}$ become functions of $\omega$ only. That is:

$$
\begin{aligned}
& 2 M W_{1}\left(v, q^{2}\right)=F_{1}(\omega) \\
& v W_{2}\left(v, q^{2}\right)=F_{2}(\omega)
\end{aligned}
$$

It is this property that is referred to as "scaling" in the variable $\omega$ in the "Bjorken limit." The variable $\mathrm{x}=\mathrm{l} / \omega$ came into use soon after the first inelastic measurements; we will use both in this paper.

Since $W_{1}$ and $W_{2}$ are related by

$$
v W_{2} / W_{1}=(1+R) /(1 / v+\omega /(2 M))
$$

it can be seen that scaling in $W$, accompanies scaling in $v W_{2}$ only if $R$ has the proper functional form to make the right hand side of the equation a function of $\omega$. In the Bjorken limit, it is evident that the ratio $v W_{2} / W_{1}$ will scale if $R$ is constant or is a function of $\omega$ only.

\section{Radiative Corrections}

Radiative corrections must be applied to the measured cross sections to eliminate the effects of the radiation of photons by electrons which occurs during the nucleon scattering itself and during traversals of material before and after scattering. These corrections also remove higher order electrodynamic contributions to the electron-photon vertex and the photon propagator. Radiative corrections as extensive as were required in the proposed scattering program had been little studied previously (Reference 38). Friedman (Reference 39), in 1959 had calculated the elements of the required "triangle," discussed in more detail below, in carrying out corrections to the inelastic scattering of $175 \mathrm{MeV}$ electrons from deuterium. Isabelle and Kendall (Reference 40), studying the inelastic scattering of electrons of

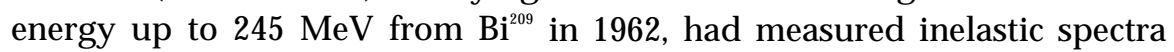
over a number of triangles and had developed the computer procedures necessary to permit computation of the corrections. These studies provided 


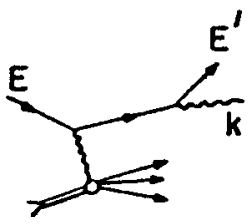

(a)

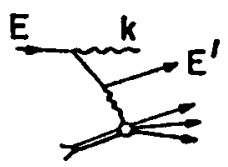

(b)

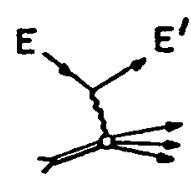

(c)

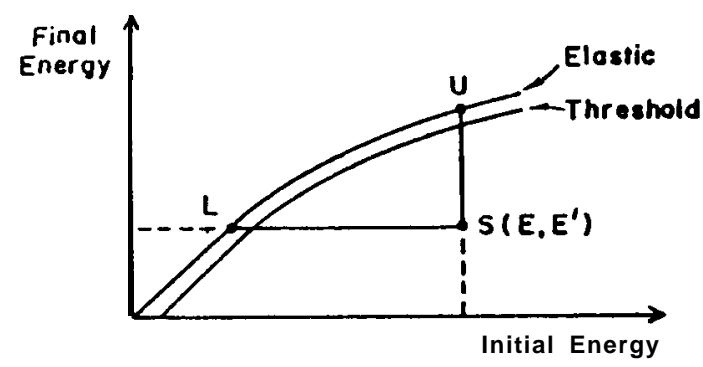

(d)

Fig. 6. Diagrams showing radiation in electron scattering (a) after exchange of a virtual photon (b) before exchange of a virtual photon. Figure $(6 \mathrm{c})$ is the diagram with radiative effects removed. Figure $(6 \mathrm{~d})$ is the kinematic plane relevant to the radiative corrections program. The text contains a further discussion of corrections procedures. A "triangle" as discussed in the text is formed by points $\mathrm{L}, \mathrm{U}$, and $\mathrm{S}$.

confidence that the procedures were tractable and the resulting errors of acceptable magnitude.

The largest correction has to be made for the radiation during scattering, described by diagrams (a) and (b) in Figure 6. A photon of energy $k$ is emitted in (a) after the virtual photon is exchanged, and in (b) before the exchange. Diagram (c) is the cross section which is to be recovered after appropriate corrections for (a) and (b) have been made. A measured cross section at fixed $E, \mathrm{E}^{1}$, and $\theta$ will have contributions from (a) and (b) for all values of $k$ which are kinematically allowed. The lowest value of $k$ is zero, and the largest occurs in (b) for elastic scattering of the virtual electron from the target particle. Thus, to correct a measured cross section at given values of $E$ and $E$, one must know the cross section over a range of incident and scattered energies.

To an excellent approximation, the information necessary to correct a cross section at an angle $\theta$ may all be gathered at the same value of $\theta$. Diagram (d) of Figure 6 shows the kinematic range in $E$ and $\mathrm{E}^{1}$ of cross sections which can contribute by radiative processes to the fundamental cross section sought at point $\mathrm{S}$, for fixed $\theta$. The range is the same for contributions from bremsstrahlung processes of the incident and scattered electrons. For single hard photon emission, the cross section at point $\mathrm{S}$ will 
have contributions from elastic scattering at points $U$ and $L$, and from inelastic scattering along the lines SL and SU, starting at inelastic threshold. If two or more photons are radiated, contributions can arise from line LU and the inelastic region bounded by lines SL and SU. The cross sections needed for these corrections must themselves have been corrected for radiative effects. However, if uncorrected cross sections are available over the whole of the "triangle" LUS, then a one-pass radiative correction procedure may be employed, assuming the peaking approximation (Reference 41), which will produce the approximately corrected cross sections over the entire triangle, including the point $\mathrm{S}$.

The application of radiative corrections required the solution of another difficulty, as it was generally not possible to take measurements sufficiently closely spaced in the E-E' plane to apply them directly. Typically live to ten spectra, each for a different $E$, were taken to determine the cross sections over a "triangle." Interpolation methods had to be developed to supply the missing cross sections and had to be tested to show that they were not the source of unexpected error. Figure 7 shows the triangles, and the locations of the spectra, for data taken in one of the experiments in the program.

In the procedures that were employed, the radiative tails from elastic electron-proton scattering were subtracted from the measured spectra before the interpolations were carried out. In the MIT-SLAC radiative correction procedures, the radiative tails from elastic scattering were calculated using the formula of Tsai (Reference 42), which is exact to lowest order in $\boldsymbol{a}$. The calculation of the tail included the effects of radiative energy degradation of the incident and final electrons, the contributions of multiple photon processes, and radiation from the recoiling proton. After the subtraction of the elastic peak's radiative tail, the inelastic radiative tails were removed in a one-pass unfolding procedure as outlined above. The particular form of the peaking approximation used was determined from a lit to an exact calculation of the inelastic tail to lowest order which incorporated a model that approximated the experimental cross sections. One set of formulas and procedures are described by Miller et al. (Reference 43) and were employed in the SLAC analysis. The measured cross sections were also corrected in a separate analysis, carried out at MIT, using a somewhat different set of approximations (Reference 44). Comparisons of the two gave corrected cross sections which agreed to within a few percent. Reference 45 contains a complete description of the MIT radiative corrections procedures that were applied, the cross checks that were carried out, and the assessment of errors arising both from the radiative corrections and from other sources of uncertainty in the experiment. Figure 8 shows the relative magnitude of the radiative corrections as a function of $W$ for a typical spectrum with a hydrogen target. While radiative corrections were the largest corrections to the data, and involved a considerable amount of computation, they were understood to a confidence level of $5 \%$ to $10 \%$ and did not significantly increase the total error in the measurements. 



Fig. 7. Inelastic measurements: where spectra were taken to determine "triangles" employed in making radiative corrections for three angles selected for some of the later experiments. The solid curves represent the kinematics of elastic electron-proton scattering. 


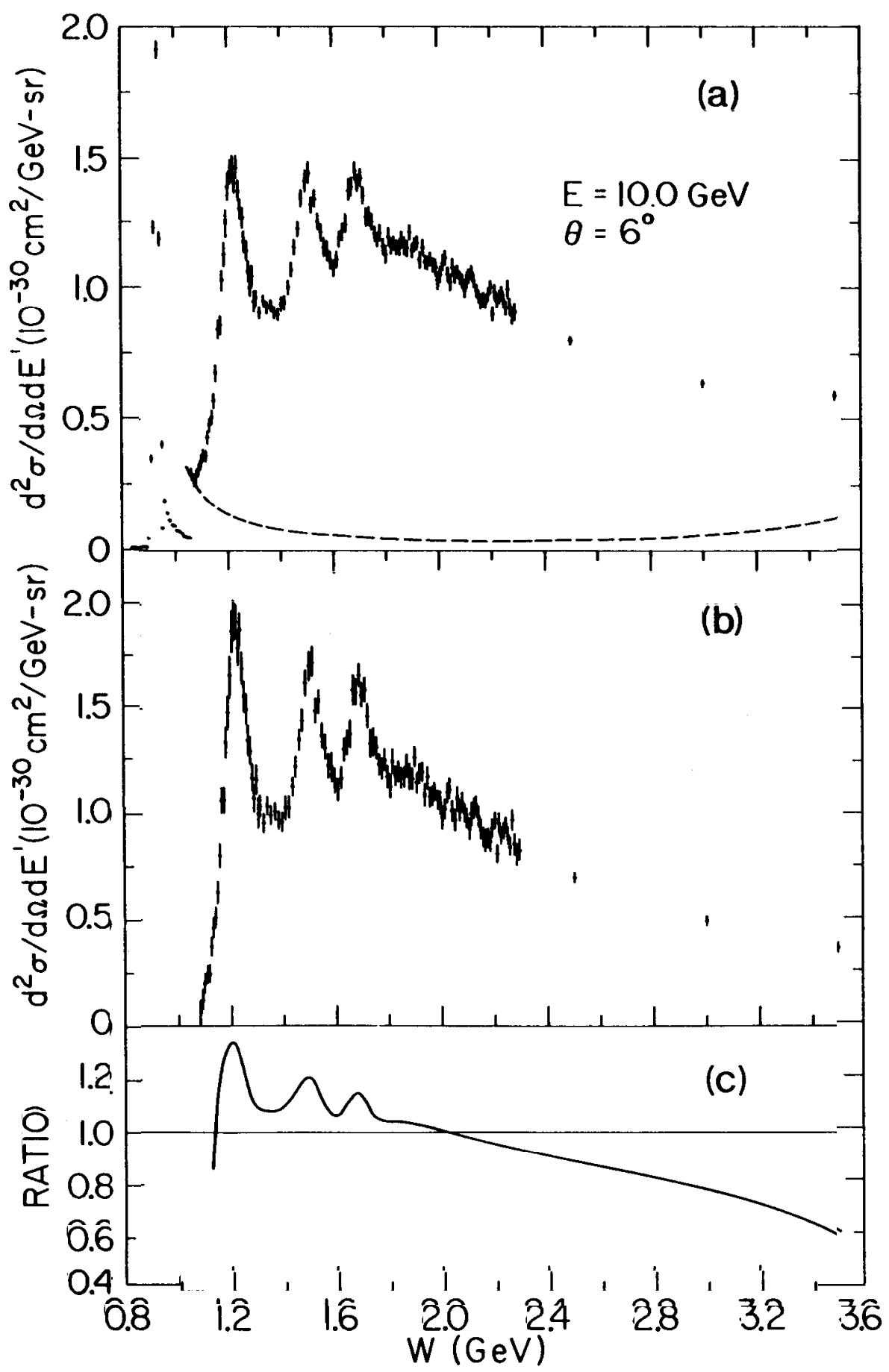

Fig. 8. Spectra of $10 \mathrm{GeV}$ electrons scattered from hydrogen at $\mathbf{6}^{\mathbf{0}}$, as a function of the final hadronic state energy W. Figure (8a) shows the spectrum before radiative corrections. The elastic peak has been reduced in scale by a factor of 8.5. The computed radiative "tail" from the elastic peak is shown. Figure (8 b) shows the same spectrum with the elastic peak's tail subtracted and inelastic corrections applied. Figure (8c) shows the ratio of the inelastic spectrum before, to the spectrum after, radiative corrections. 




Fig. 9. Spectra of electrons scattered from hydrogen at $q^{2}$ up to $4(\mathrm{GeV} / \mathrm{c})^{2}$. The curve for $q^{2}=0$ represents an extrapolation to $q^{2}=0$ of electron scattering data acquired at $\boldsymbol{\theta}=1.5^{\circ}$. Elastic peaks have been subtracted and radiative corrections have been applied. 


\section{Electron Proton Scattering: Results.}

The scattered electron spectra observed in the experiments had a number of features whose prominence depended on the initial and final electron energies and the scattering angle. At low $q^{2}$ both the elastic peak and resonance excitations were large, with little background from non-resonant continuum scattering either in the resonance region or at higher missing masses. As $q^{2}$ increased, the elastic and resonance cross sections decreased rapidly, with the continuum scattering becoming more and more dominant. Figure 9 shows four spectra of differing $q^{2}$. Data points taken at the elastic peak and in the resonance region were closely spaced in $E^{\prime}$ so as to allow fits to be made to the resonance yields, but much larger steps were employed for larger excitation energies.

Figures $10 \mathrm{a}$ and $10 \mathrm{~b}$ show visual fits to spectra over a wide range in energy and scattering angle (including one spectrum from the accelerator at the Deutsches Electronen Synchrotron (DESY)), illustrating the points discussed above.

Two features of the non-resonant inelastic scattering that appeared in the first continuum measurements were unexpected. The first was a quite weak $q^{2}$ dependence of the scattering at constant $W$. Examples for $W=2.0$ and $W$ $=3.0 \mathrm{GeV}$, taken from data of the first experiment, are shown in Figure 11 as a function of $\mathrm{q}^{2}$. For comparison the $\mathrm{q}^{2}$ dependence of elastic scattering is shown also.

The second feature was the phenomenon of scaling. During the analysis of the inelastic data, J. D. Bjorken suggested a study to determine if $\nu W_{2}$ was a function of $\boldsymbol{\omega}$ alone. Figure 12a shows the earliest data so studied: $W_{2}$, for six values of $q^{2}$, as a function of $v$. Figure $12 b$ shows $F_{2}=v W_{2}$ for 10 values of $\mathrm{q}^{2}$, plotted against $\boldsymbol{\omega}$. Because $\mathrm{R}$ was at that time unknown, $\boldsymbol{F}_{\mathbf{2}}$ was shown for the limiting assumptions, $R=0$ and $R=$ co. It was immediately clear that the Bjorken scaling hypothesis was, to a good approximation, correct. This author, who was carrying out this part of the analysis at the time, recalls wondering how Balmer may have felt when he saw, for the first time, the striking agreement of the formula that bears his name with the measured wavelengths of the atomic spectra of hydrogen.

More data showed that, at least in the first regions studied and within sometimes large errors, scaling held nearly quantitatively. As we shall see, scaling holds over a substantial portion of the ranges of $v$ and $q^{2}$ that have been studied. Indeed the earliest inelastic e-p experiments (Reference 30) showed that approximate scaling behavior occurs already at surprisingly non-asymptotic values of $q^{2} \geq 1.0 \mathrm{GeV}^{2}$ and $W \geq 2.6 \mathrm{GeV}$.

The question quickly arose as to whether there were other scaling variables that converged to $\boldsymbol{\omega}$ in the Bjorken limit, and that provided scaling behavior over a larger region in $v$ and $q^{2}$ than did the use of $\boldsymbol{\omega}$. Several were proposed (Reference 46) before the advent of QCD, but because this theory predicts small departures from scaling, the search for such variables was abandoned soon after. 


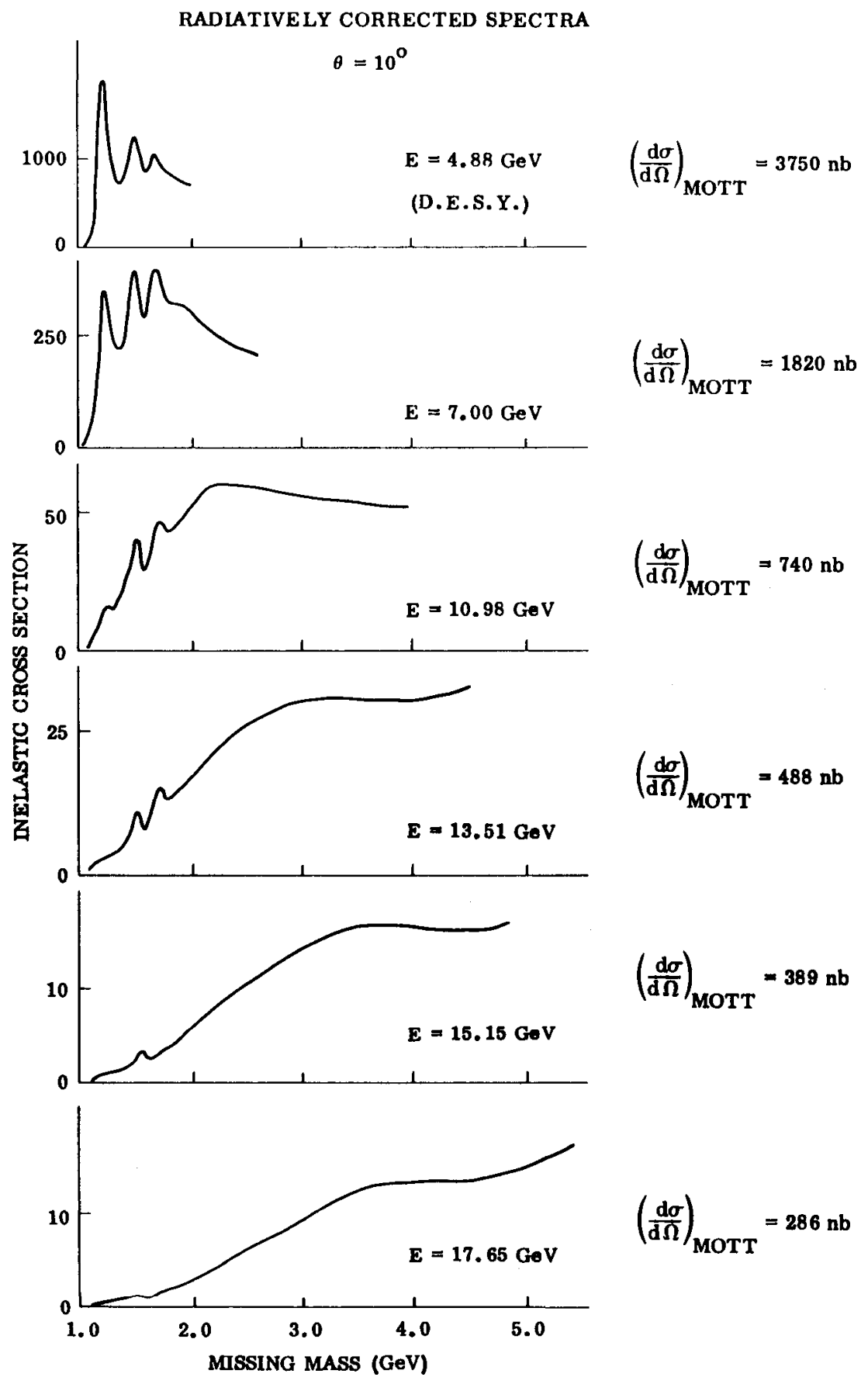

Fig. 10a. Visual fits to spectra showing the scattering of electrons from hydrogen at $10^{\circ}$ for primary energies, $E$, from $4.88 \mathrm{GeV}$ to $17.5 \mathrm{GeV}$. The elastic peaks have been subtracted and radiative corrections applied. The cross sections are expressed in nanobarns per GeV per steradian. The spectrum for $\mathrm{E}=4.88 \mathrm{GeV}$ was taken at DESY; W. Bartel, et al., Phys. Lett., B28 148 (1968). 


\section{RADIATIVELY CORRECTED CROSS SECTIONS \\ $E \approx 13.5 \mathrm{GeV}$}

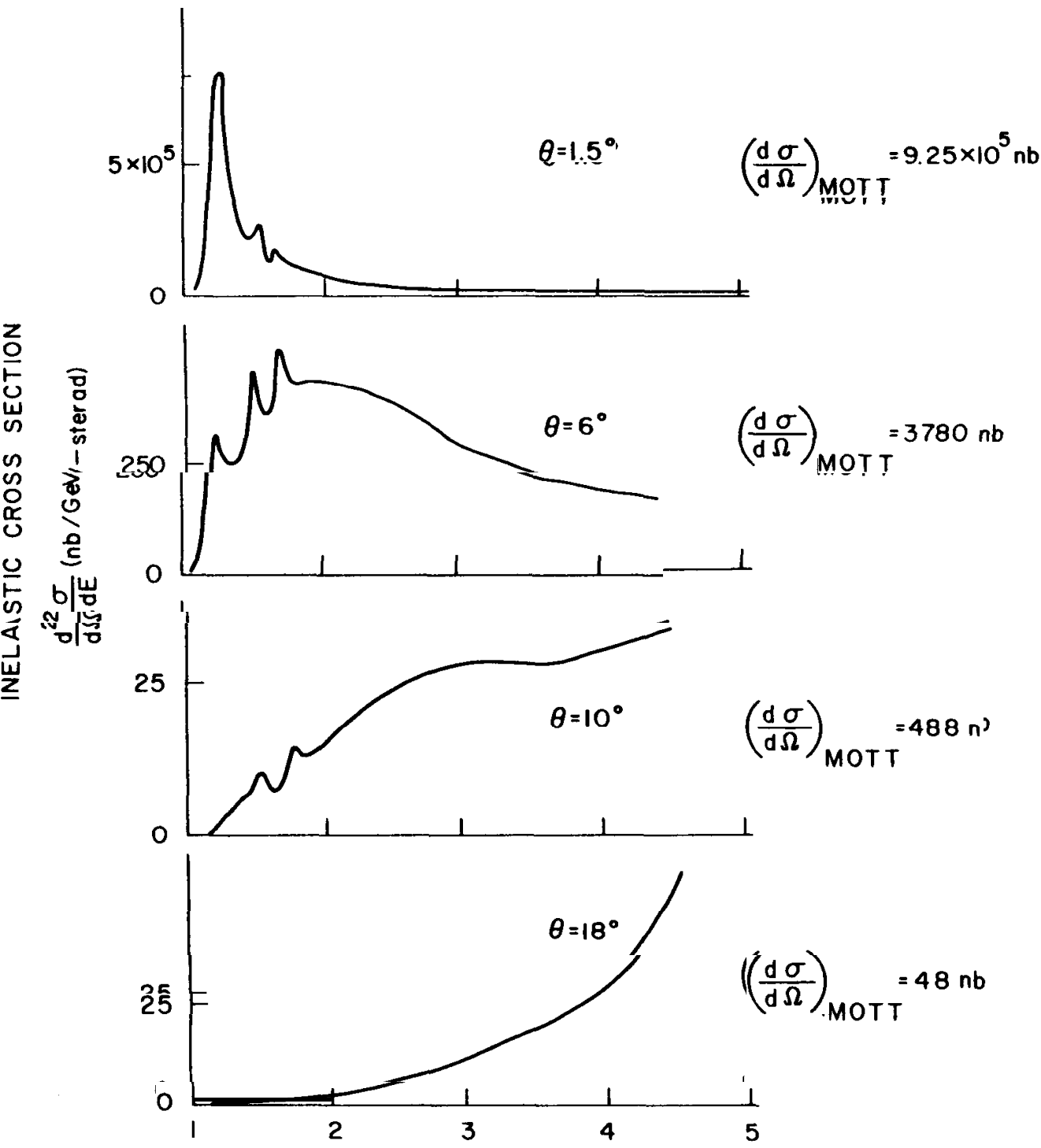

MISSING MASS W(GeV)

Fig. $1 O b$. Visual fits to spectra showing the scattering of electrons from hydrogen at a primary energy $\mathrm{E}$ of approximately $13.5 \mathrm{GeV}$, for scattering angles from $1.5^{\circ}$ to $18^{\circ}$. The $1.5^{\circ}$ curve is taken from MIT-SLAC data used to obtain photoabsorption cross sections. 


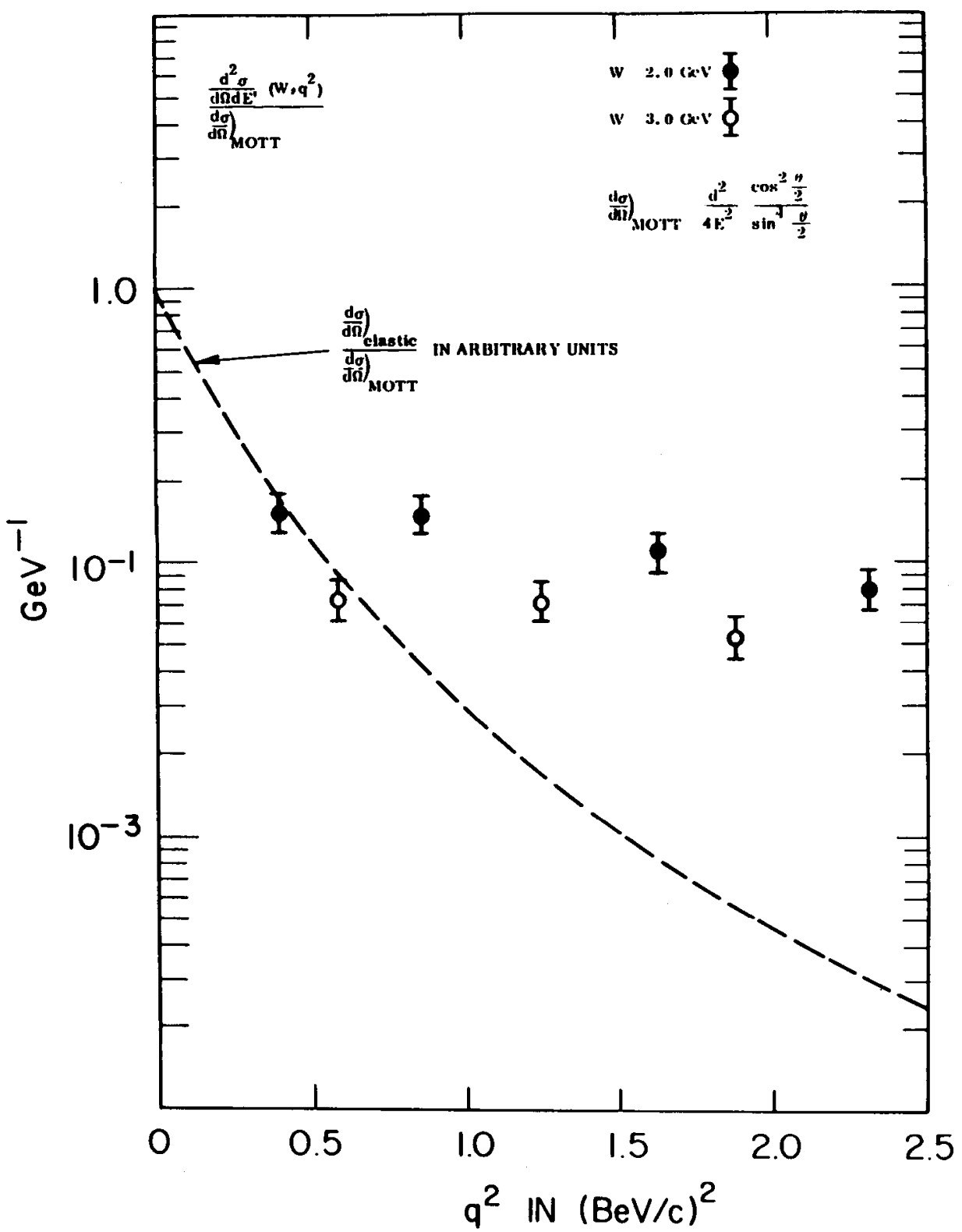

Fig. 11. Inelastic data for $W=2$ and $3 \mathrm{GeV}$ as a function of $q^{2}$. This was one of the earliest examples of the relatively large cross sections and weak $q^{2}$ dependence that were later found to characterize the deep inelastic scattering and which suggested point-like nucleon constituents. The $\mathrm{q}^{2}$ dependence of elastic scattering is shown also; these cross sections have been divided by $\sigma_{\mathrm{M}}$ 



0.10

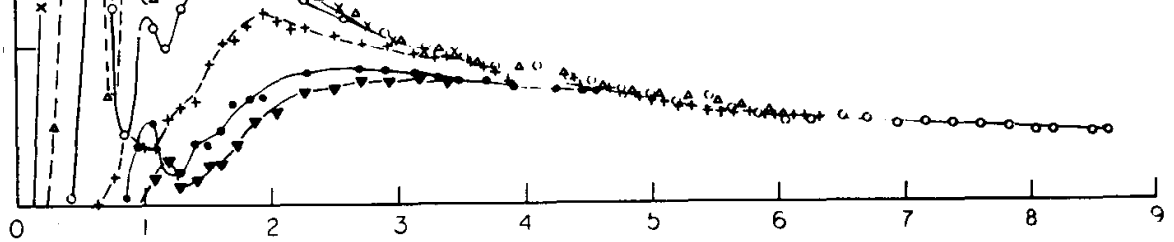

ENEERGYY LôŜSS $\ddot{\nu}$ iñ Bèv

b:

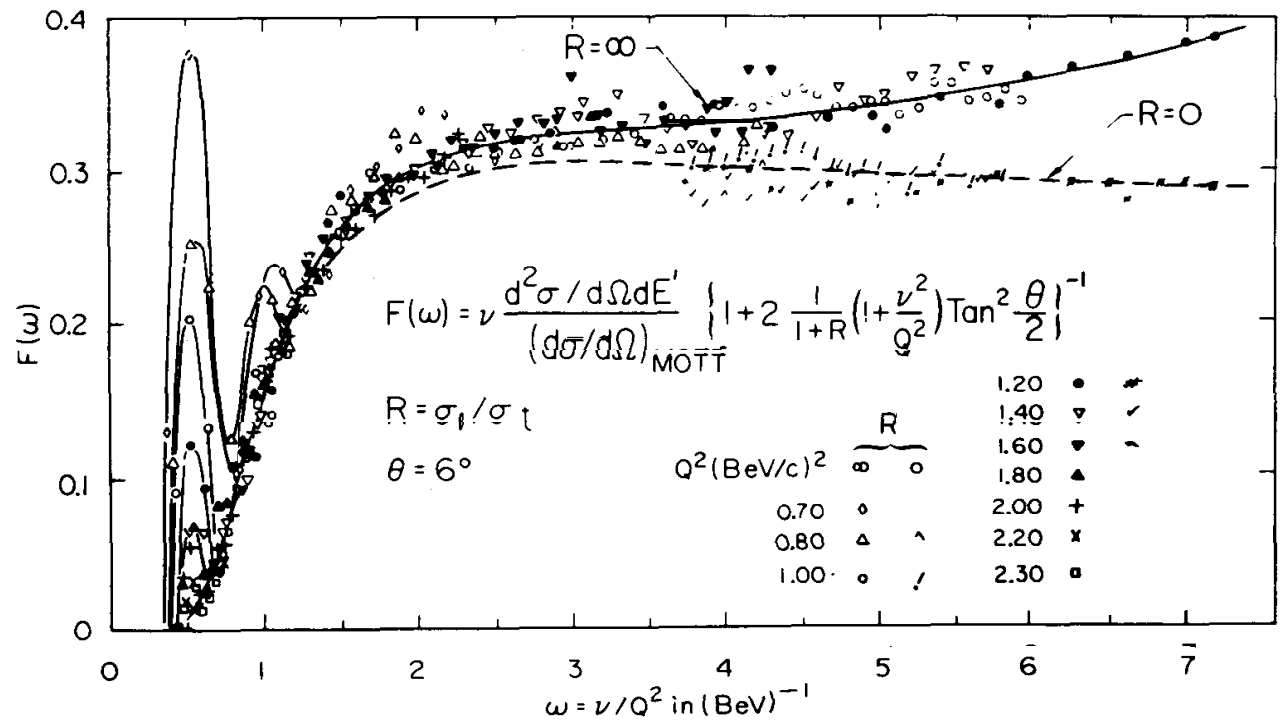

Fig. 12. (a) The inelastic structure function $W_{2}\left(v, q^{2}\right)$ plotted against the electron energy loss $v$. (b) The quantity $F_{1}=v W_{2}(\omega)$. The "nesting" of the data observed here was the first evidence of scaling. The figure is discussed further in the text. 


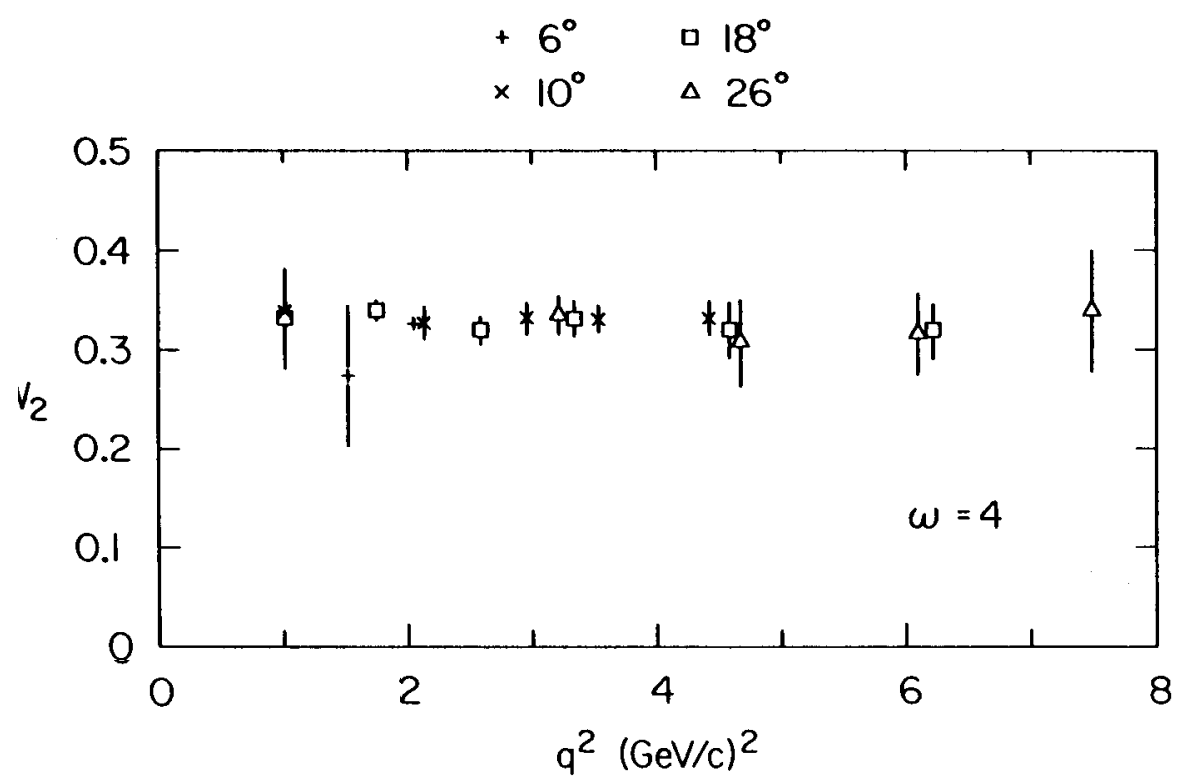

Fig. 13. An early öbservation of scäling: $\bar{v} W_{2}^{-}$for the proton as a function of ${ }^{2}{ }^{2}$ for $W>2 \mathrm{GeV}$, at $\omega=4$.

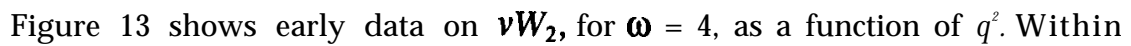
the errors there was no $q^{2}$ dependence.

A more complex separation procedure was required to determine $R$ and the structure functions, as discussed above. The kinematic region in $q^{2}-W^{2}$ space available for the separation is shown in Figure 14. This figure also shows the 75 kinematic points where, after the majority of the experiments were complete, separations had been made. Figure 15 displays sample leastsquare fits to $\boldsymbol{\Sigma}\left(\boldsymbol{v}, q^{2}, \boldsymbol{\theta}\right)$ vs $\boldsymbol{\varepsilon}\left(\boldsymbol{v}, q^{2}, \boldsymbol{\theta}\right)$, as defined earlier, in comparison with data, from which $\boldsymbol{\sigma}_{\mathrm{L}}$ and $\boldsymbol{\sigma}_{\mathrm{T}}$ and then $\mathrm{R}$, were found.

A rough evaluation of scaling is provided by, for example, inspecting a plot of the data taken by the collaboration on $v W_{2}$ against $x$ as shown in Figure 16. These data, to a fair approximation, describe a single function of $x$. Some deviations, referred to as scale breaking, are observed. They are more easily inspected by displaying the $q^{2}$ dependence of the structure functions. Figure 17 shows separated values of $2 M W_{1}$ and $\nu W_{2}$ from data taken late in the program, plotted against $q^{2}$ for a series of constant values of $x$. With extended kinematic coverage and with smaller experimental errors, sizeable scale breaking was observed in the data.

\section{Theoretical Implications of the Electron-Proton Inelastic Scattering Data.}

As noted earlier, the discovery, during the first inelastic proton measurements, of the weak $q^{2}$ dependence of the structure function $v W_{2}$, coupled with the scaling concept inferred from current algebra and its roots in the quark theory, at once suggested new possibilities concerning nucleon structure. At the 1968 Vienna Meeting, where the results were made public for 


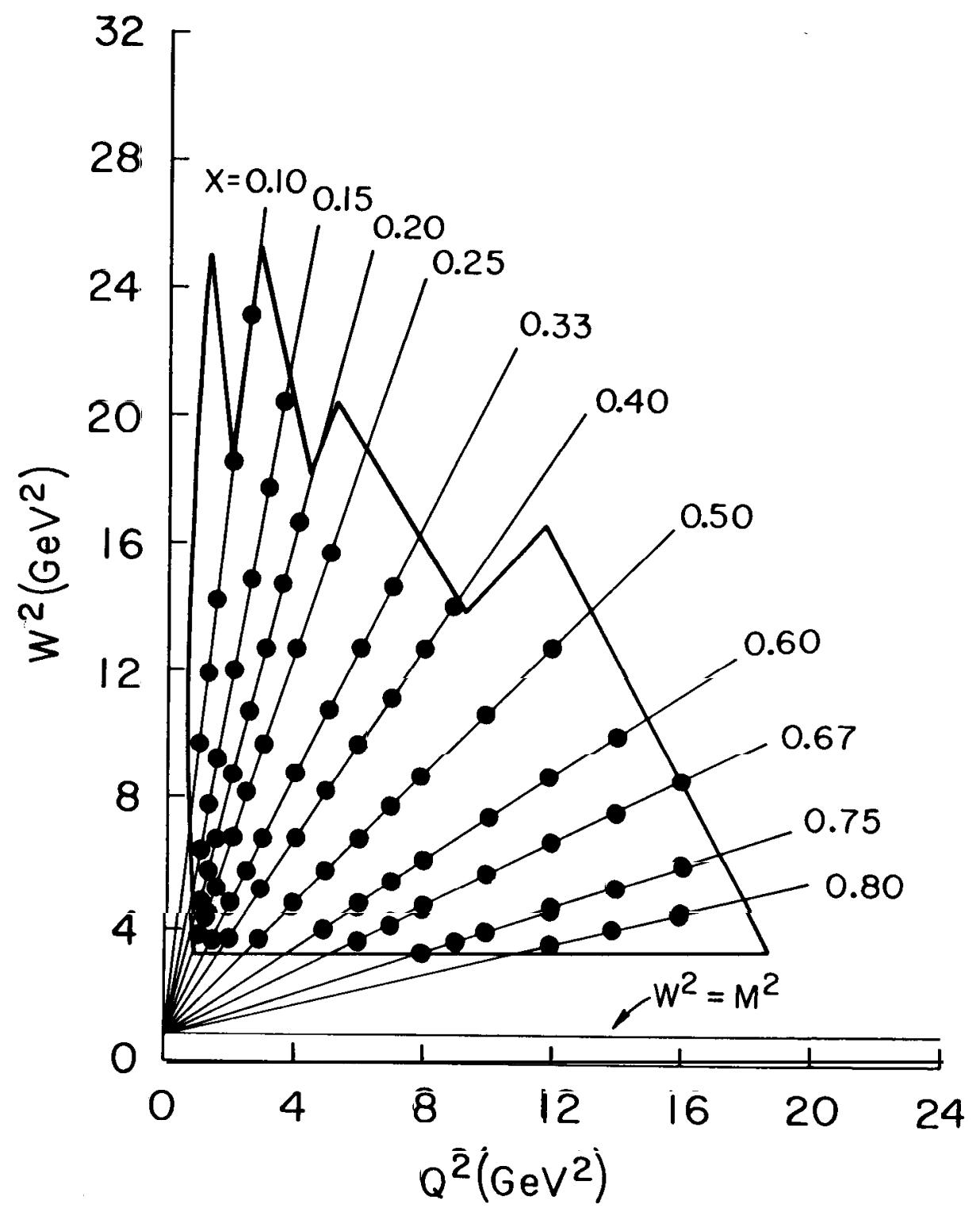

Fig. 14. The kinematic region in $q^{2}-W^{2}$ space available for the extraction of $\mathbf{R}$ and the structure functions. Separations were made at the 75 kinematic points $\left(v, q^{2}\right)$ shown. 

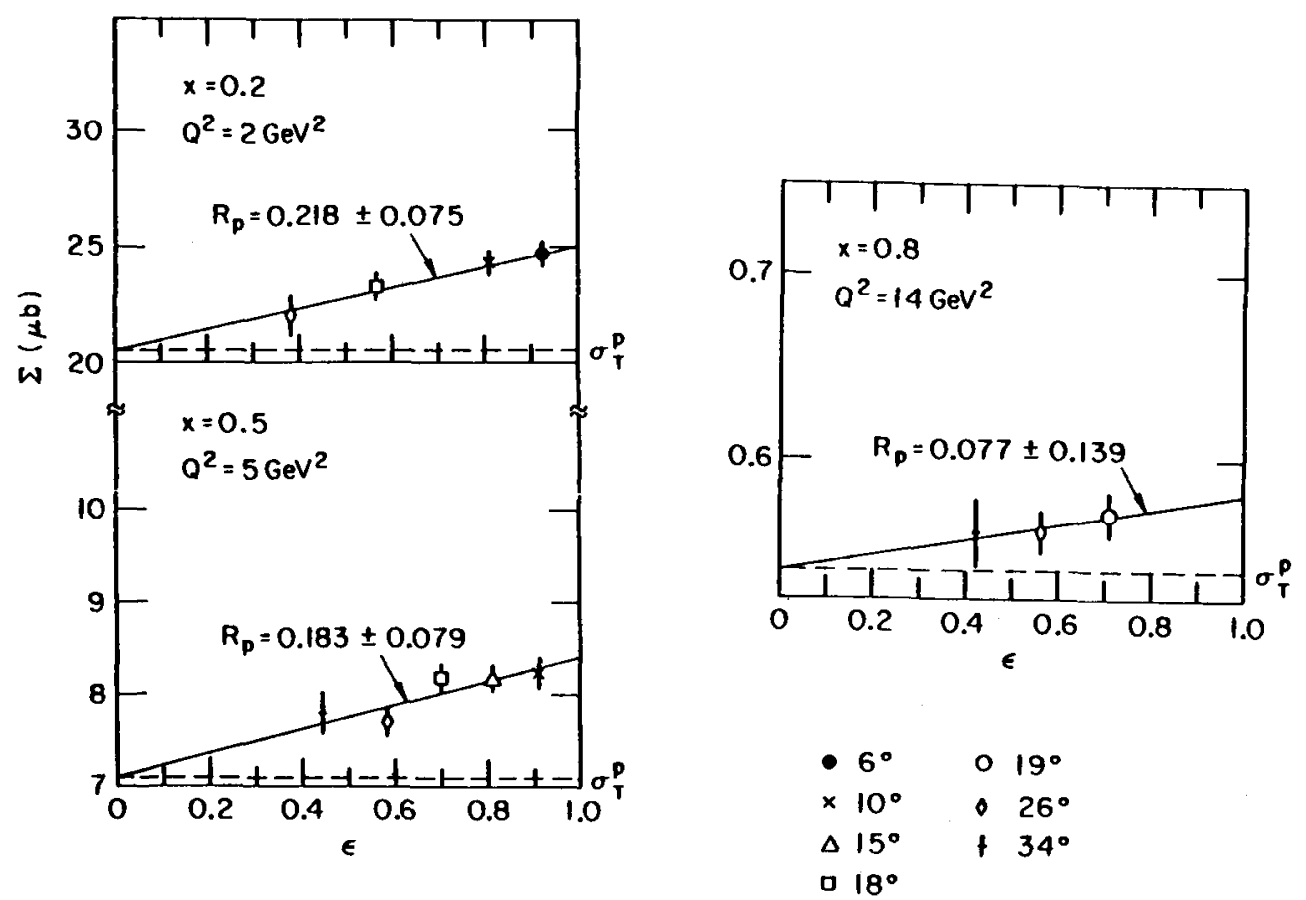

Fig. 15. Sample least-square fits to $\Sigma$ vs $\varepsilon$ in comparison with data from the proton. The quantities $\mathrm{R}$ and $\boldsymbol{\sigma}_{\mathrm{T}}$ were available from the fitting parameters, and from them $\sigma_{\mathrm{L}}$ was determined.

the first time, the rapporteur, W. K. H. Panofsky, summed up the conclusions (Reference 47): “Therefore theoretical speculations are focussed on the possibility that these data might give evidence on the behavior of pointlike, charged structures within the nucleon."

Theoretical interest at SLAC in the implications of the inelastic scattering increased substantially after an August 1968 visit by R. P. Feynman. He had been trying to understand hadron-hadron interactions at high energy assuming constituents he referred to as partons. On becoming aware of the inelastic electron scattering data, he immediately saw in partons an explanation both of scaling and the weak $q^{2}$ dependence. In his initial formulation (Reference 48), now called the naive parton theory, he assumed that the proton was composed of point-like partons, from which the electrons scattered incoherently. The model assumed an infinite momentum frame of reference, in which the relativistic time dilation slowed down the motion of the constituents. The transverse momentum was neglected, a simplification relaxed in later elaborations. The partons were assumed not to interact with one another while the virtual photon was exchanged: the impulse approximation of quantum mechanics. Thus, in this theory, electrons scattered from constituents that were "free," and therefore the scattering reflected the properties and motions of the constituents. This assumption of a nearvanishing of the par-ton-parton interaction during lepton scattering, in the 

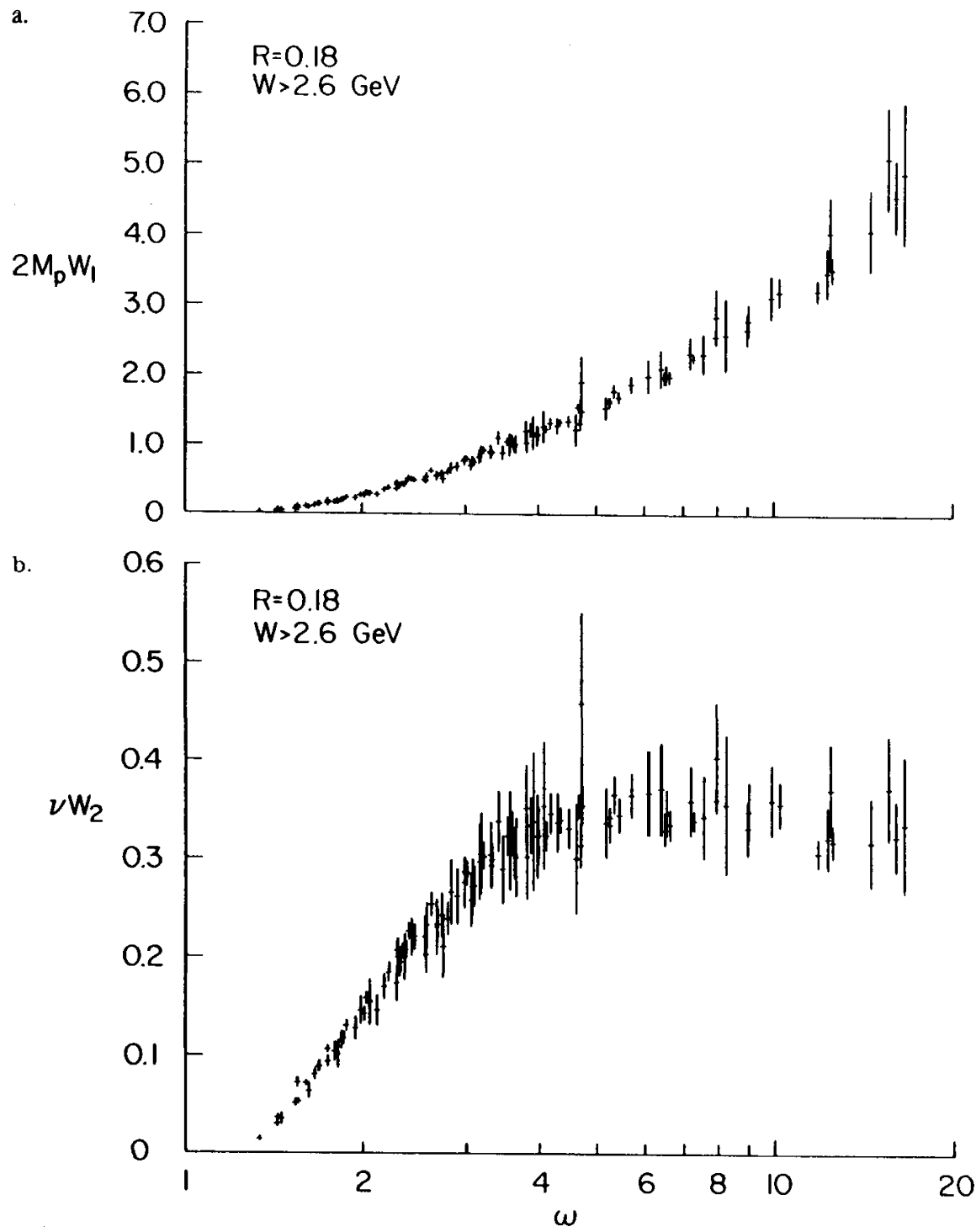

Fig. 16. (a,b) Scaling: $\left.I F_{1}=2 M W_{1}(\omega)\right) v s\left(\omega\right.$, and $\left.F_{2}=W W W_{2}(\omega)\right) W(\omega$, forr the proten.

Bjorken limit, was subsequently shown to be a consequence of QCD known as asymptotic freedom. Feynman came to Stanford again, in October 1968, and gave the first public talk on his parton theory, stimulating much of the theoretical work which ultimately led to the identification of his partons with quarks.

In November 1968, Curt Callan and David Gross (Reference 49) showed that R, given in Equation (6), depended on the spins of the constituents in a parton model and that its kinematic variation constituted an important test 


$$
\text { a. }
$$

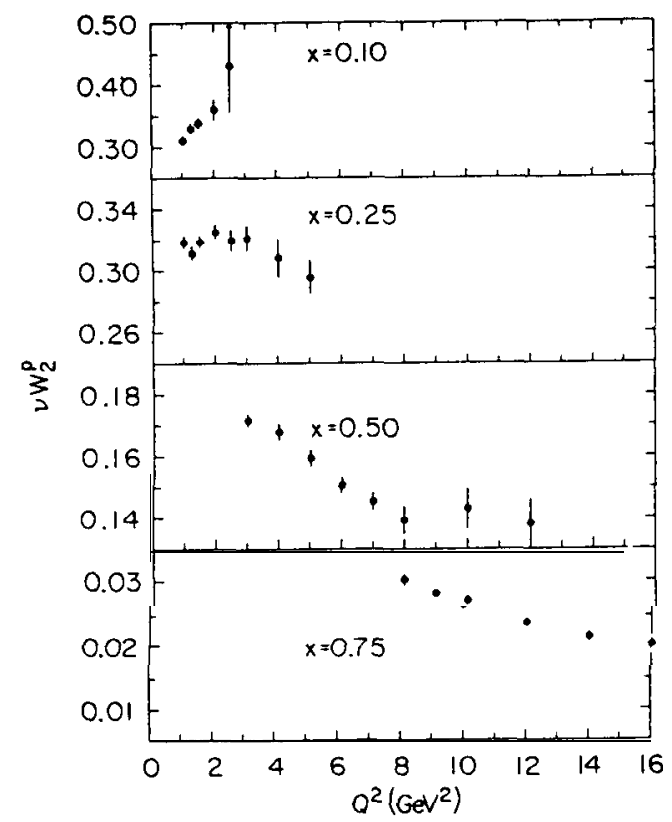

b.

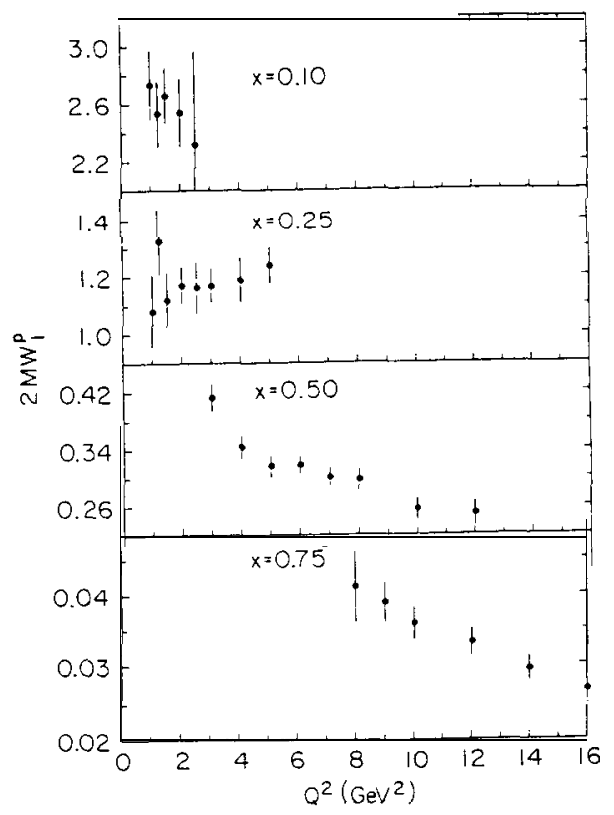

Fig. 17. (a,b) $F_{1}$ and $F_{2}$ as functions of $q^{2}$, for fixed values of $x$, for the proton

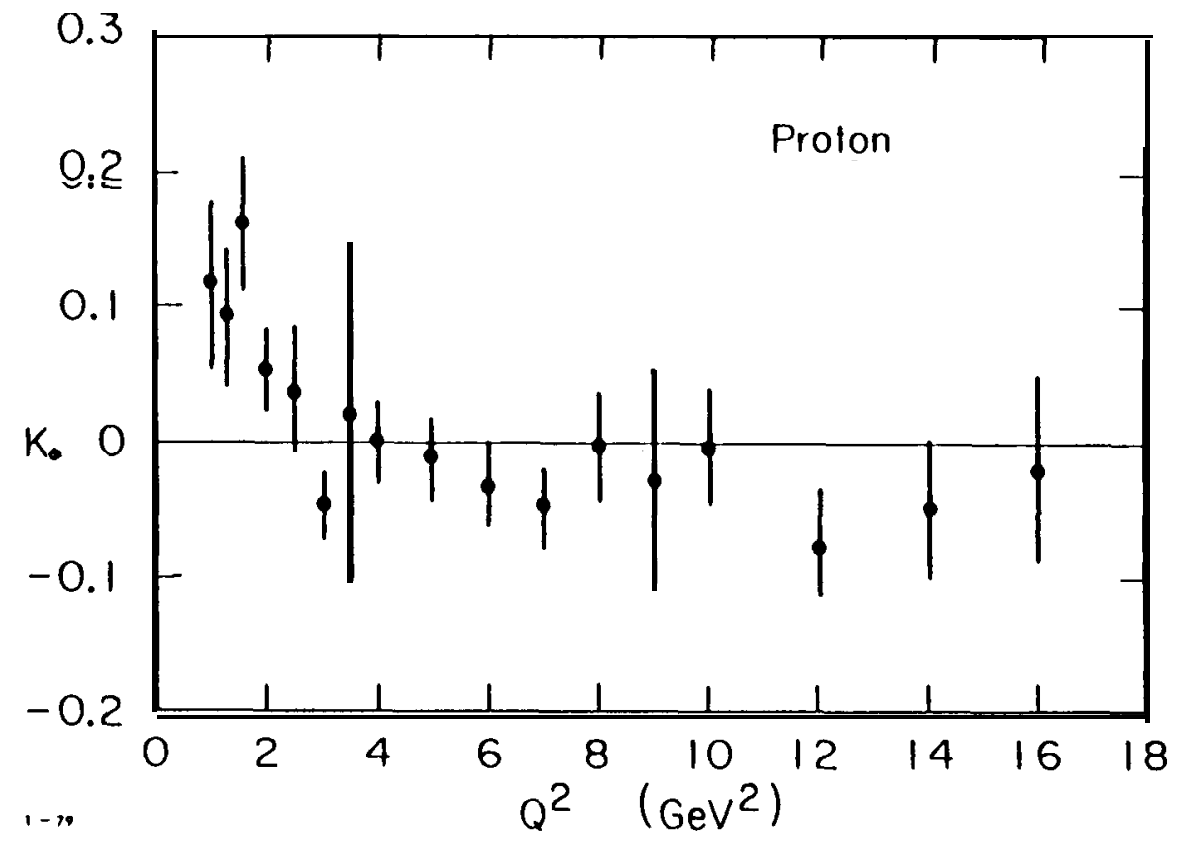

Fig. 18. The Callan-Gross relation: $K_{0} v s q^{2}$, where $K_{0}$ is defined in the text. These results established the spin of the partons as $\mathrm{I} / 2$. 
of such models. For spin I/2, $R$ was expected to be small, and, for the naive parton model, where the constituents are assumed unbound in the Bjorken limit, $R=q^{2} / v^{2}$ (ie, $F_{2}=x F_{1}$ ). More generally, for spin I/2 partons, $R=$ $g(x)\left(q^{2} / v^{2}\right)$. This is equivalent to the scaling of $v R$.

Spin zero or one partons led to the prediction $R \neq 0$ in the Bjorken limit, and would indicate that the proton cloud contains elementary bosons. Small values of $R$ were found in the experiment and these were totally incompatible with the predictions of Vector Meson Dominance. Later theoretical studies (Reference 50 ) showed that deviations from the general CallanG ross rule would be expected at low $\mathbf{x}$ and low $q^{2}$. A direct evaluation of the Callan-Gross relation for the naive parton model may be found from

$$
K_{0}=F_{2} /\left(x F_{1}\right)-1
$$

which vanishes when the relation is satisfied. $K_{0}$ is shown in Figure 18 , as a function of $q^{2}$. Aside from the expected deviations at low $q^{2}, K_{0}$ is consistent with zero, establishing the parton spin as $\mathrm{I} / 2$.

\section{Epilogue}

After the initial inelastic measurements were completed, deuteron studies were initiated to make neutron structure functions accessible. Experiments were made over a greater angular range and statistical, radiative, and systematic errors were reduced. The structure functions for the neutron were found to differ from the proton's. Vector Meson Dominance was abandoned and by 1972 all diffractive models, and nuclear democracy, were found to be inconsistent with the experimental results. Increasingly detailed parton calculations and sum rule comparisons, now focussing on quark constituents, required sea quarks - virtual quark-antiquark pairs - in the nucleon, and, later, gluons - neutral bosons that provided the inter-quark binding.

On the theoretical front, a special class of theories was found that could incorporate asymptotic freedom and yet was compatible with the binding necessary to have stable nucleons. N eutrino measurements confirmed the spin I/2 assignment for partons and that they had fractional, rather than integral electric charge. The number of "valence" quarks was found to be 3, consistent with the original 1964 assumptions.

By 1973, the picture of the nucleon had clarified to such an extent that it became possible to construct a comprehensive theory of quarks and gluons and their strong interactions: QCD. This theory was built on the concept of "color," whose introduction years before (Reference 51) made the nucleons' multi-quark wave functions compatible with the Pauli principle, and, on the assumption that only "color-neutral" states exist in nature, explained the absence of all unobserved multi-quark configurations (such as quark-quark and quark-quark-antiquark) in the known array of hadrons. Furthermore, as noted earlier, QCD was shown to be asymptotically free (Reference 52). 
By that year the quark-parton model, as it was usually called, satisfactorily explained electron-nucleon and neutrino-nucleon interactions, and provided a rough explanation for the very high energy "hard" nucleon-nucleon scattering that had only recently been observed. The experimenters were seeing quark-quark collisions.

By the end of the decade, the fate of quarks recoiling within the nucleon in high energy collisions had been understood; for example, after quark pair production in electron-positron colliders, they materialized as back-toback jets composed of ordinary hadrons (mainly pions), with the angular distributions characteristic of spin I/2 objects. G luon-jet enhancement of quark jets was predicted and then observed, having the appropriate angular distributions for the spin 1 they were assigned within QCD. Theorists had also begun to deal, with some success, with the problem of how quarks remained confined in stable hadrons.

Quantum Chromodynamics describes the strong interactions of the hadrons and so can account, in principle at least, for their ground state properties as well as hadron-hadron scattering. The hadronic weak and electromagnetic interactions are well described by electroweak theory, itself developed in the late 1960s. The picture of the nucleon, and the other hadrons, as diffuse, structureless objects was gone for good, replaced by a successful, nearly complete theory.

\section{ACKNOWLEDGEMENTS}

There were many individuals who made essential contributions to this work. An extensive set of acknowledgements is given in Reference 53.

\section{REFERENCES}

1. R.E.Taylor, Deep Inelastic Scattering: The Early Years. Les Prix Nobel 1990. Hereafter "Taylor."

2. J. I. Friedman, Deep Inelastic Scattering: Comparisons with the Quark Model. Les Prix Nobel 1990. Hereafter "Friedman."

3. S. C. Frautschi, Regge Poles and S-Matrix Theory, W. A. Benjamin (1963). Hereafter "Frautschi."

4. G. F. Chew and S. C. Frautschi, Phys. Rev. Lett. 8, 394 (1961).

5. P. D. B. Collins and E. J. Squires, Regge Poles in Particle Physics, Springer-Verlag, Berlin (1968). For a broad review of the strong interaction physics of the period see Martin L. Perl, High Energy Hadron Physics, John Wiley \& Sons, (N ew York 1974). See also Frautschi.

6. G. F. Chew, S. C. Frautschi, and S. Mandelstam, Phys. Rev. 126, 1202 (1962).

7. G. Veneziano, Nuovo Cim. 57A, 190 (1968). See also J. H. Schwarz, Phys. Rep. 8, 269 (1973).

8. J.J.Sakurai, Phys. Rev. Lett. 22, 981 (1969).

9. M. Cell-Mann, Phys. Lett. 8, 214 (1964).

10. G. Zweig, CERN-8182/ Th.401 (Jan. 1964) and CERN-8419/ Th.412 (Feb. 1964), both unpublished. Hereafter "Zweig."

11. M. Gell-Mann, C. I. T. Synchrotron Lab. Rep't, CTSL-20 (unpublished) and Y. Ne'eman, Nuc. Phys. 26, 222 (1961). See also M. Gell-Mann and Y. Ne'eman, The Eightfold Way, W. A. Benjamin (1964). 
12. The quark model explained why triplet, sextet, and 27-plets of then-current SU (3) were absent of hadrons. With rough quark mass assignments, it could account for observed mass splittings within multiplets, and it provided an understanding of the anomalously long lifetime of the phi meson (discussed later in this paper).

13. Lawrence W. Jones, Rev. Mod. Phys. 49, 717 (1977).

14. "...we know that...[mesons and baryons] are mostly, if not entirely, made up out of one another. . . The probability that a meson consists of a real quark pair rather than two mesons or a baryon and antibaryon must be quite small." M. Cell-Mann, Proc.XIII Ih Inter. Conf. on High En. Phys., Berkeley, California 1967.

15. "A dditional data is necessary and very welcome in order to destroy the picture of elementary constituents." J. D. Bjorken. "I think Prof. Bjorken and I constructed the sum rules in the hope of destroying the quark model." Kurt Gottfried. Both quotations from Proc. 1967 Internat. Symp. on Electron and Photon Interac. at High Energy, Stanford, California, Sept. 5 -9, 1967.

16. R. Dalitz, Session 10, Rapporteur. Proc. XIII Inter. Conf: High En. Phys., Berkeley, 1966. (University of California, Berkeley).

17. C. Becchi and G. Morpurgo, Phys. Lett. 17, 352 (1965).

18. K. Gottfried, Phys. Rev. Lett. 18, 1174 (1967).

19. Zweig believed from the outset that the nucleon was composed of "physical" quark constituents. This was based primarily on his study of the properties of the phi meson. It did not decay rapidly to $p$ - $\pi$ as expected but rather decayed roughly two orders of magnitude slower to kaon-antikaon, whose combined mass was near the threshold for the decay. He saw this as a dynamical effect; one not explainable by selection rules based on the symmetry groups and explainable only by a constituent picture in which the initial quarks would "flow smoothly" into the final state. He was "severely criticized" for his views, in the period before the MIT-SLAC results were obtained. Private communication, February 1991.

20. According to a popular book on the quark search, Zweig, a junior theorist visiting at CERN when he proposed his quark theory, could not get a paper published describing his ideas until the middle 1970s, well after the constituent model was on relatively strong ground; M. Riordan, The Hunting of the Quark, Simon and Schuster, N ew York (1987). His preprints (cf. Zweig) did, however, reach many in the physics community and helped stimulate the early quark searches.

21. A. Pais, Inward Bound, Oxford University Press, New York City (1986).

22. "Throughout the 1960s, into the 1970s, papers, reviews, and books were replete with caveats about the existence of quarks." Andrew Pickering, Constructing Quarks, University of Chicago Press (Chicago 1984).

23. Further discussion of sum rules and their comparisons with data is to be found in Friedman.

24. "Such particles [quarks] presumably are not real but we may use them in our field theory anyway." Physics 1, 63 (1964).

25. "N ow what is going on? What are these quarks? It is possible that real quarks exist, but if so they have a high threshold for copious production, many BeV; ..." Proc. XIII Inter. Conf. on High En. Phys., Berkeley, California, 1967.

26. "We shall find these results [sum rules requiring cross sections of order Rutherford scattering from a point particle] so perspicuous that, by an appeal to history, an interpretation in terms of 'elementary constituents' of the nucleon is suggested." He pointed out that high energy lepton-nucleon scattering could resolve the question of their existence and noted that "it will be of interest to look at very large inelasticity and dispose, without ambiguity, of the model completely." "Current Algebra at Small Distances," lecture given at International School of Physics, "Enrico Fermi," XLI Course, Varenna, Italy, July 
1967. SLAC Pub. 338, August 1967 (unpublished) and Proceedings of the International School of Physics "Enrico Fermi", Course XLI: Selected Topics in Particle Physics, J. Steinberger, ed. (A cademic Press, N ew York, 1968).

27. T. D. Lee: "I'm certainly not the person to defend the quark models, but it seems to me that at the moment the assumption of an unsubtracted dispersion relation [the subject of the discussion] is as ad hoc as the quark model. Therefore, instead of subtracting the quark model one may also subtract the unsubtracted dispersion relation." J. Bjorken: "I certainly agree. I would like to dissociate myself a little bit from this as a test of the quark model. I brought it in mainly as a desperate attempt to interpret the rather striking phenomena of a point-like behavior. One has this very strong inequality on the integral over the scattering cross section. It's only in trying to interpret how that inequality could be satisfied that the quarks were brought in. There may be many other ways of interpreting it." Discussion in Proc. 1967 Internat. Symp. on Electron and Photon Interac. at High Energy, Stanford, Sept 5 -9, 1967.

28. Proposal for Spectrometer Facilities at SLAC, submitted by SLAC Groups A and C, and physicists from MIT and CIT. (Stanford, California, undated, unpublished). Proposal for Initial Electron Scattering Experiments Using the SLAC Spectrometer Facilities: Proposal $4 b$ "The Electron-Proton Inelastic Scattering Experiment," submitted by the SLAC-MIT-CIT Collaboration, 1 January 1966 (unpublished).

29. The Stanford Two-Mile Accelerator R. B. Neal, General Editor, W. A. Benjamin (N ew York 1968).

30. 14th Int. Conf. High Energy Phys., Vienna, Aug., 1968. E. D. Bloom et al., Phys. Rev. Lett. 23, 930 (1969); M. Breidenbach et al., ibid 23, 935 (1969).

31. W. R. Ditzler, et al., Phys. Lett. 57B, 201 (1975).

32. L. W. Whitlow, et al., Phys. Lett. B250, 193 (1990) and L. W. Whitlow, SLAC Report 357, March 1990 (unpublished).).

33. M. Rosenbluth, Phys. Rev. 79, 615 (1950).

34. P. N. Kirk, et al., Phys. Rev. D8, 63 (1973).

35. S. D. Drell and J. D. Walecka, A nn. Phys. 28, 18 (1964).

36. L. Hand, Phys. Rev. 129, 1834 (1963).

37. J. D. Bjorken, Phys. Rev. 179, 1547 (1969). Although the conjecture was published after the experimental results established the existence of scaling, the proposal that this might be true was made prior to the measurements as discussed later in the text.

38. J. D. Bjorken, Ann. Phys. 24, 201 (1963).

39. J. I. Friedman, Phys. Rev. 116, 1257 (1959).

40. D. Isabelle and H. W. Kendall, Bull. Am. Phys. Soc. 9, 95 (1964). The final report on the experiment is S. Klawansky et al., Phys. Rev. C7, 795 (1973).

41. G. Miller et al., Phys. Rev. D5, 528 (1972); L. W. Mo and Y. S. Tsai, Rev. Mod. Phys. 41, 205 (1969).

42. Y. S. Tsai, Proc. Nucleon Struct. Conf. Stanford 1963, p. 22 1. Stanford Univ. Press (Stanford 1964), edited by R. Hofstadter and L. I. Schiff.

43. G. Miller et al., Phys. Rev. D5, 528 (1972).

44. Poucher, J. S. 1971. PhD thesis, MIT, (unpublished).

45. A. Bodek et al., Phys. Rev. D20, 1471 (1979).

46. J. I. Friedman and H. W. Kendall, Ann. Rev. Nuc. Sci., 22, 203 (1972). A portion of this publication is used in the present paper.

47. W.K.H.Panofsky, in 14th Int. Conf. High Energy Phys., Vienna, Aug., 1968, edited by J. Prentki and J. Steinberger (CERN, Geneva), p 23.

48. R. P. Feynman, Phys. Rev. Lett. 23, 1415 (1969).

49. C. Callan and D. J. Gross, Phys. Rev. Lett. 21, 311 (1968).

50. R. P. Feynman, Photon-Ha\&on Interactions, W. A. Benjamin (1972).

51. M. Y. Han and Y. Nambu, Phys. Rev. 139B, 1006 (1965). 
52. D. Gross and F. Wilczek, Phys. Rev. Lett. 30, 1343 (1973), D. Politzer, ibid., 1346.

53. J. I. Friedman, H. W. Kendall, and R. E. Taylor, Deep Inelastic Scattering: Acknowledgements, Les Prix Nobel 1990. 